Article

\title{
On the Role of a ZDDP in the Tribological Performance of Femtosecond Laser-Induced Periodic Surface Structures on Titanium Alloy against Different Counterbody Materials
}

\author{
Jon Joseba Ayerdi ${ }^{1,2, *} \mathbb{1}$, Nadine Slachciak ${ }^{2}$, Iñigo Llavori ${ }^{1}{ }^{\circledR}$, Alaitz Zabala ${ }^{1}{ }^{1}$, \\ Andrea Aginagalde ${ }^{1}$, Jörn Bonse ${ }^{2}$ (i) and Dirk Spaltmann ${ }^{2}$ \\ 1 Surface Technologies, Mondragon University, Faculty of Engineering, Loramendi 4, 20500 \\ Arrasate-Mondragon, Spain \\ 2 Bundesanstalt für Materialforschung und -prüfung (BAM), Unter den Eichen 87, 12205 Berlin, Germany \\ * Correspondence: jon.ayerdig@alumni.mondragon.edu
}

Received: 3 July 2019; Accepted: 30 August 2019; Published: 3 September 2019

check for updates

\begin{abstract}
Laser-induced periodic surface structures (LIPSS, ripples) with $\sim 500-700 \mathrm{~nm}$ period were produced on titanium alloy (Ti6Al4V) surfaces upon scan processing in air by a Ti:sapphire femtosecond laser. The tribological performance of the surfaces were qualified in linear reciprocating sliding tribological tests against balls made of different materials using different oil-based lubricants. The corresponding wear tracks were characterized by optical and scanning electron microscopy and confocal profilometry. Extending our previous work, we studied the admixture of the additive 2-ethylhexyl-zinc-dithiophosphate to a base oil containing only anti-oxidants and temperature stabilizers. The presence of this additive along with the variation of the chemical composition of the counterbodies allows us to explore the synergy of the additive with the laser-oxidized nanostructures.
\end{abstract}

Keywords: lubricant additives; laser-induced periodic surface structures (LIPSS); wear; friction

\section{Introduction}

The material Ti6Al4V is one of the most widely used titanium alloys (e.g., in the aerospace industry and for implants in medicine) that are usually restricted in tribological applications due to the low surface hardness, the rather high coefficient of friction and the low abrasive wear. By means of surface modification technologies, the aforementioned shortcoming can be overcome. One of the most promising approaches is based on the use of lasers, which allow a contactless, fast, and reliable surface functionalization.

Ultrafast laser processing can be used to generate various surface morphologies, employing either direct contour-shaping or, for larger surface areas, functionalization via the generation of "self-organized" micro- and nanostructures [1]. This allows researchers to induce different surface functionalities in the fields of optics [2], fluidics [3], medicine [4,5], and tribology [6] and can be scaled up to the current demands for sizes and production rates in industrial applications.

The exposure to high-intensity pulsed laser radiation excites the treated materials into extreme conditions, which then return to equilibrium through various relaxation channels. Potentially, these involve phase transitions such as melting, evaporation (ablation), recrystallization, or accompanying chemical alterations in the ambient atmosphere (e.g., oxidation), etc., finally featuring different types of surface topographies [7]. One specific kind of these topographies currently gaining attention in various fields of applications is called laser-induced periodic surface structures (LIPSS, ripples). They are formed either perpendicular or parallel to the linear laser beam polarization and usually exhibit spatial 
periods in the sub-micrometer range [8]. These laser-induced nanostructures can be produced in a very reliable manner in a contactless single-step approach.

In a series of previous publications, we have explored the tribological performance of two different types of these surface nanostructures, high spatial frequency LIPSS (HSFL) [9] and low spatial frequency LIPSS (LSFL) $[6,10,11]$, in the regime of mixed friction, reporting for the LSFL a remarkably positive effect on the coefficient of friction $(\mathrm{CoF})$ and the associated wear of LIPSS-covered titanium or titanium alloy surfaces. They were tested in linear reciprocating sliding tribological tests (RSTT) in a fully formulated engine oil against a ball of hardened 100Cr6 steel and compared to polished reference surfaces. Via contrasting juxtaposition to identical RSTT conducted in non-additivated paraffin oil, it was speculated that this positive effect may be attributed to the presence of the additive (zinc dialkyl dithiophosphate, ZDDP [12]) contained in the engine oil (VP1) [10], able to efficiently bond to the laser-structured but not to the polished surfaces. These ZDDP molecules could then cover the laser-treated surfaces and, therefore, prevent a direct contact of the tested metals.

Apart from the topographic alterations, spatially and depth-resolved chemical analyses also revealed a fs-laser-induced oxidation process during the LIPSS formation on titanium [13]. In comparative oxidation experiments (thermal oxidation vs. anodic oxidation vs. LIPSS-induced oxidation) a beneficial tribological effect was found only for sufficiently large oxide layer thicknesses $(>150 \mathrm{~nm})$ and for high temperature surface oxidation [14]. The graded oxide layer present for the LSFL consisting mainly of amorphous $\mathrm{TiO}_{2}$ and micro-crystalline $\mathrm{Ti}_{2} \mathrm{O}_{3}$ [13] may help to stabilize mechanically the oxygen containing near the surface region, preventing its delamination during the tribological demands/stresses. Moreover, with some hundreds of nanometers, the ball-sample deformation during the RSTT is of similar magnitude as the surface modulation depth of the LSFL [10], allowing a confinement of the lubricant in the tribological contact area during the RSTT.

In this work, we test our previous hypothesis that an additivated, fully formulated engine oil in combination with a laser-oxidized surface is beneficial for the tribological performance of $\mathrm{Ti}$ surfaces [10]. Specific emphasis is laid on the disclosure of the relevance of some specific anti-wear additives on the tribological performance of fs-laser-processed titanium alloy (Ti6Al4V) surfaces. By replacing the commercial engine oil (VP1) by an admixture of its base oil (VPX) with a single additive-here, the anti-wear additive (2-ethylhexyl zinc dithiophosphate)—its crucial role can be specifically addressed under our standard tribological testing conditions. Moreover, since the oils and additives are optimized for metallic (steel) surfaces, by varying the counterbody material, the role of the surface chemistry is further elucidated. Despite the use of engine oil, this work is an academic study and not one aimed at a specific engineering problem.

\section{Experimental}

Commercial grade-5 titanium alloy (Ti6Al4V) was purchased from Schumacher Titan GmbH (Solingen, Germany). The rods of $25 \mathrm{~mm}$ diameter were cut into circular slabs of $8 \mathrm{~mm}$ thickness. The top surfaces of the slabs were subsequently mechanically polished resulting in surface roughness parameters $R_{\mathrm{a}}=5 \mathrm{~nm}$ and $R_{\mathrm{rms}}=6 \mathrm{~nm}$.

For large area surface processing, a linearly polarized commercial Ti:sapphire laser amplifier system was used (Femtolasers, Compact Pro, Vienna, Austria: $\tau=30 \mathrm{fs}$ pulse duration, $\lambda=790 \mathrm{~nm}$ central wavelength, $v=1 \mathrm{kHz}$ pulse repetition rate). The titanium alloy samples were mounted on a motorized $x-y-z$ linear translation stage and placed perpendicular to the incident laser beam, realizing at the surface a Gaussian-like beam profile with a radius of $w_{0}\left(1 / e^{2}\right) \sim 140 \mu \mathrm{m}$. Square-shaped areas of $5 \times 5 \mathrm{~mm}^{2}$ were processed upon meandering movement of the sample $\left(v_{\mathrm{x}}=5 \mathrm{~mm} / \mathrm{s} \mathrm{scan}\right.$ velocity, $\Delta y=0.1 \mathrm{~mm}$ line-offset) under the focused laser beam at $(35 \pm 2) \mu \mathrm{J} /$ pulse. These laser-processing conditions correspond to a laser peak fluence of $\varphi_{0} \sim 0.11 \mathrm{~J} / \mathrm{cm}^{2}$ in front of the surface and an effective number of laser pulses per beam spot diameter of $N_{\text {eff }} \sim 56$ in the direction of the processed lines. Once processed, the samples were ultrasonically cleaned in acetone for $5 \mathrm{~min}$. All samples were stored in a desiccator (up to a few months prior to the laser irradiation or the surface characterizations 
following the tribological tests). Given the very high temperatures of the titanium surface transiently exceeding $5000 \mathrm{~K}$ (estimated in [13]), repetitively reached during the fs-laser irradiation process, a significant storage-related post-oxidation of the laser treated samples at room temperature cannot be expected here.

The characteristics of the surface ripples were previously studied in detail on an identically processed sample revealing low-spatial frequency LIPSS (LSFL) with periodicities of $(620 \pm 80) \mathrm{nm}$ along with height modulation depths of $\pm 150 \mathrm{~nm}$ [10], typically manifested in a sine-like surface modulation. The modulation depths of the LSFL are similar to the ball-sample deformation during the RSTT (see Table 1). This may help to confine the lubricant in the tribological contact area of the nanostructured sample surface. For further details on the fs-laser processing and sample characterization, the reader is referred to [10].

RSTT measuring the coefficient of friction by the dissipated energy method were conducted with an in house-built tribometer [15] by sliding the LSFL-covered samples against either a hardened and polished steel ball (100Cr6, $\varnothing=10 \mathrm{~mm}, R_{\mathrm{a}}=8 \mathrm{~nm}$ ), a polished polycrystalline aluminium oxide ball $\left(\mathrm{Al}_{2} \mathrm{O}_{3}, \varnothing=10 \mathrm{~mm}, R_{\mathrm{a}}=10 \mathrm{~nm}\right)$, or a polished polycrystalline silicon nitride ball $\left(\mathrm{Si}_{3} \mathrm{~N}_{4}, \varnothing=10 \mathrm{~mm}\right.$, $\left.R_{\mathrm{a}}=4 \mathrm{~nm}\right)$ as counterbodies. The normal load applied by dead weight is so small $(1 \mathrm{~N})$ that the stiffness of the holders is not compromised. The tribological tests were carried out in mixed lubrication conditions to have both, the effects of the laser-modified surface and the applied oil involved. The range of uncertainty in the friction coefficient measurements is \pm 0.02 . Due to the limited availability of fs-laser-structured samples, each test was done once. The same RSTT conditions as in [10] were used in order to have directly comparable results. All relevant experimental parameters for the RSTT are summarized in Table 1.

Table 1. Linear reciprocating sliding tribological test conditions.

\begin{tabular}{|c|c|c|c|}
\hline Test Parameter [Unit] & \multicolumn{3}{|c|}{ Value } \\
\hline Tribosystem & \multicolumn{3}{|c|}{$\begin{array}{l}\text { Linear reciprocating sliding tribological test (RSTT) } \\
\left.\qquad F_{n}\right\rfloor\end{array}$} \\
\hline Samples & \multicolumn{3}{|c|}{ fs-LIPSS (LSFL) on Ti6Al4V } \\
\hline Counterbodies & \multirow{2}{*}{\multicolumn{3}{|c|}{$\begin{array}{c}100 \mathrm{Cr} 6 / \mathrm{Al}_{2} \mathrm{O}_{3} / \mathrm{Si}_{3} \mathrm{~N}_{4} \text { balls of } 10 \mathrm{~mm} \text { diameter } \\
\text { 100Cr6: 200/ } \mathrm{Al}_{2} \mathrm{O}_{3}: \sim 365 / \mathrm{Si}_{3} \mathrm{~N}_{4}: \sim 300 / \text { Ti6Al4V: } \sim 100\end{array}$}} \\
\hline Young's Modulus [GPa] & & & \\
\hline Hardness [GPa] & \multirow{2}{*}{\multicolumn{3}{|c|}{$\begin{array}{c}100 \mathrm{Cr} 6: \sim 7 / \mathrm{Al}_{2} \mathrm{O}_{3}: \underset{1000}{\sim 17 / \mathrm{Si}_{3} \mathrm{~N}_{4}:} \sim 16 / \mathrm{Ti} 6 \mathrm{Al} 4 \mathrm{~V}: \sim 1 \\
\end{array}$}} \\
\hline Stroke $[\mu \mathrm{m}]$ & & & \\
\hline Frequency $[\mathrm{Hz}]$ & \multicolumn{3}{|c|}{1} \\
\hline Normal load $[\mathrm{N}]$ & \multicolumn{3}{|c|}{1} \\
\hline Number of cycles & \multicolumn{3}{|c|}{1000} \\
\hline Atmosphere & \multicolumn{3}{|c|}{ Laboratory air } \\
\hline Temperature $\left[{ }^{\circ} \mathrm{C}\right]$ & \multicolumn{3}{|c|}{$\sim 20-22$} \\
\hline Relative humidity [\%] & \multicolumn{3}{|c|}{$\sim 22-35$} \\
\hline Lubrication & \multicolumn{3}{|c|}{$\mathrm{VPX} / \mathrm{VPX}+0.5 \%$ RC 3180} \\
\hline $\begin{array}{l}\text { Hertzian average/maximum contact } \\
\text { pressure }[\mathrm{MPa}]\end{array}$ & $\begin{array}{l}\text { vs. } 100 \mathrm{Cr} 6 \\
242 / 363\end{array}$ & vs. $\mathrm{Al}_{2} \mathrm{O}_{3}$ & vs. $\mathrm{Si}_{3} \mathrm{~N}_{4}$ \\
\hline $\begin{array}{l}\text { Hertzian radius of the sample-ball } \\
\text { contact }[\mu \mathrm{m}]\end{array}$ & $\begin{array}{l}\text { vs. } 100 \mathrm{Cr} 6 \\
36\end{array}$ & $\begin{array}{l}\text { vs. } \mathrm{Al}_{2} \mathrm{O}_{3} \\
34\end{array}$ & $\begin{array}{l}\text { vs. } \mathrm{Si}_{3} \mathrm{~N}_{4} \\
35\end{array}$ \\
\hline Hertzian sample-ball deformation $[\mu \mathrm{m}]$ & $\begin{array}{l}\text { vs. } 100 \mathrm{Cr} 6 \\
0.26\end{array}$ & $\begin{array}{l}\text { vs. } \mathrm{Al}_{2} \mathrm{O}_{3} \\
0.23\end{array}$ & $\begin{array}{l}\text { vs. } \mathrm{Si}_{3} \mathrm{~N}_{4} \\
\quad 0.24\end{array}$ \\
\hline
\end{tabular}

The tribological tests were conducted using VPX oil as base lubricant, i.e., a variant of the polyalphaolefin (PAO) based factory fill Castrol engine oil SAE 0W-30 "VP1" (Castrol, Liverpool, UK) containing only anti-oxidation and anti-corrosion additives but no friction modifiers or wear protecting constituents. As a second variant, the commercial anti-wear additive RC 3180 purchased from LANXESS Deutschland GmbH (Business Unit Rhein Chemie, Cologne, Germany) was added 
to the base oil VPX. This additive consists of 2-ethylhexyl zinc dithiophosphate containing zinc, phosphorous, and sulphur by $9.5,8$, and 16 wt $\%$, respectively. The optimum percentage of the additive content resulting in the lowest $\mathrm{CoF}$ and wear was identified as $0.5 \mathrm{wt} \% \mathrm{RC} 3180$ addition. That optimized mixture was then used for all RSTT experiments involving RC 3180-additivated VPX oil. The results for synthetic paraffin oil and for the fully additivated engine oil VP1 were already published in [10]. However, we will show them here along with the new results for direct comparison. After the RSTT, the samples were cleaned in benzene (petroleum ether) for $15 \mathrm{~min}$ using an ultrasonic bath in order to remove the residual lubricants.

The corresponding wear tracks were inspected by optical microscopy (OM, Carl Zeiss, Discovery V20/Keyence, VHX 5000, Oberkochen, Germany) and scanning electron microscopy (SEM, Carl Zeiss, Gemini Supra 40, Oberkochen, Germany). The volume of large wear scars was calculated by measuring the cross-sectional area and the width/length of the scars by means of a 3D confocal profilometer (Nanofocus, $\mu$-surf Expert, Oberhausen, Germany) and subsequently applying analytical equations following the procedure described in the ASTM D7755-11 (2017) standard [16]. However, on the LSFL-covered and not completely worn surfaces the wear volume was estimated by assuming that roughly half of the wear track area (only at the peaks of the topography and not at the valleys) was removed over the depth measured by the profilometry (i.e., a few tens to hundreds of nanometers only).

In addition, selected parts of a wear track were analysed by scanning transmission electron microscopy (STEM). The sample was prepared by a focused ion beam (FIB) milling machine (FEI, Quanta 3D, Waltham, MA, USA) using an in situ lift-out technique. The preparation of the TEM lamella involved the deposition of a protective Pt cap layer at the region of interest, before thinning it by Ga-ions up to electron-transparency. The Pt layer protects the covered sample surface from being damaged during the FIB preparation of the lamella. This lamella of $\sim 100 \mathrm{~nm}$ thickness was then characterized in a scanning transmission electron microscope (JEOL, JEM 2200FS, Akishima, Tokyo, Japan) operated at $200 \mathrm{kV}$ with a point resolution better than $0.25 \mathrm{~nm}$. The system is equipped with a field emission gun, an in-column energy filter and an energy-dispersive $X$-ray (EDX) spectroscopy system for elemental studies.

\section{Results and Discussion}

The tribological performance of the laser-processed surfaces was characterized by means of RSTT in two different lubricants (Section 3.1) and for three different counterbody materials (Section 3.2). Previous works under identical test conditions $[10,11]$ disclosed no significant dependence on the $\mathrm{CoF} /$ wear results regarding the sliding direction relative to the orientation of the LIPSS for a metallic counterbody. Thus, the current tribological tests were always carried out in the direction perpendicular to the ablation lines and additionally on a non-irradiated or polished surface (without LIPSS) as a reference. Note that due to an update of the RSTT tribometer, the data acquisition rate was improved. Hence, the CoF results (for paraffin oil and VP1 oil) published in 2014 in Ref. [10] and displayed in the following section for comparison exhibit 10 times less data points.

\subsection{Reciprocating Sliding Tribological Tests (RSTT) against 100Cr6 in Different Lubricants}

In a first set of experiments the VPX base oil was used in RSTT under identical testing conditions previously used for paraffin oil [10]. This choice of lubricant allows us to take benefit of the anti-oxidation and temperature stabilizing components contained in VPX oil, which are not present in paraffin oil. Figure 1 compiles these new RSTT results (left column) and compares them to the previous results in paraffin oil (right column). 


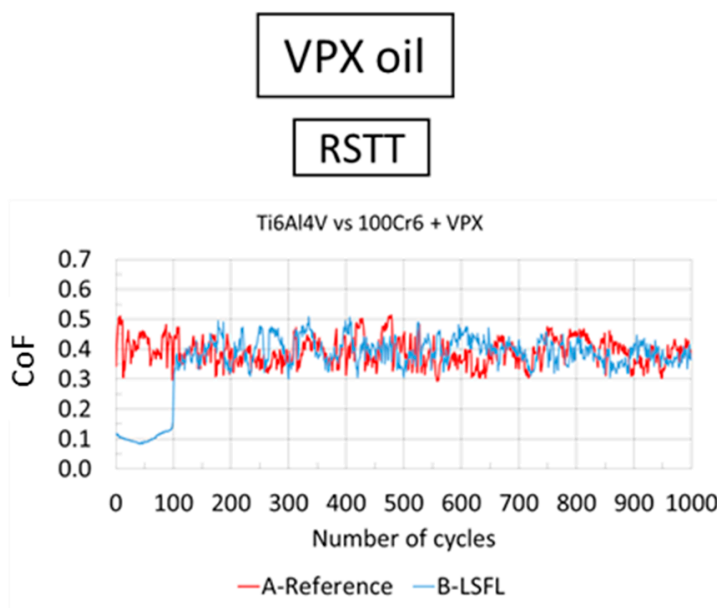

(a)

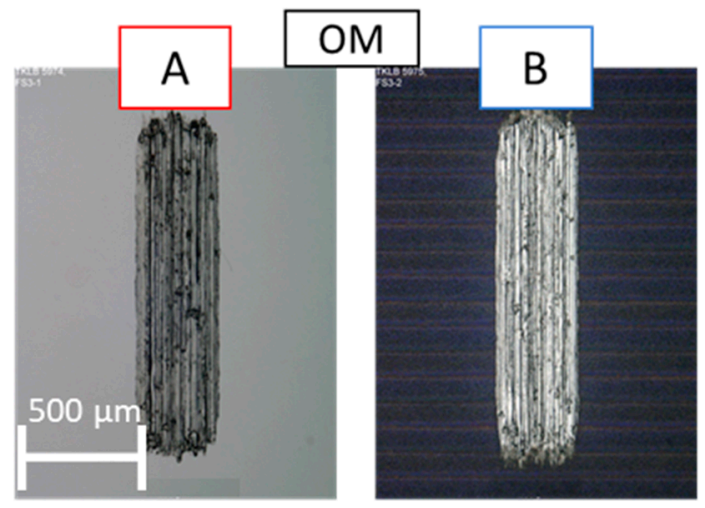

(b)

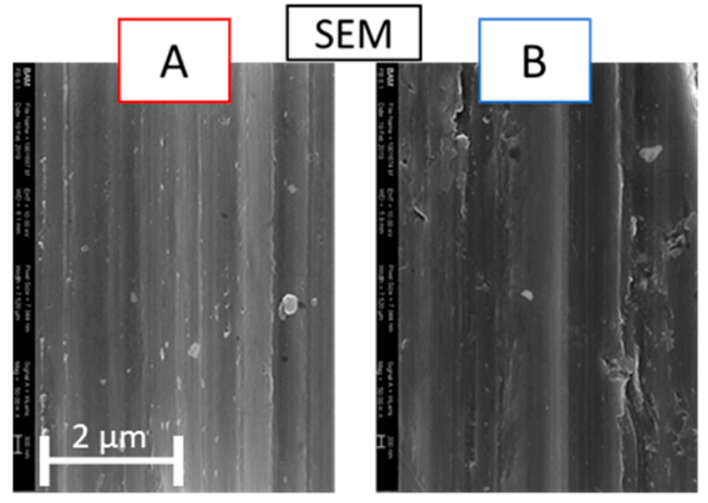

(c)

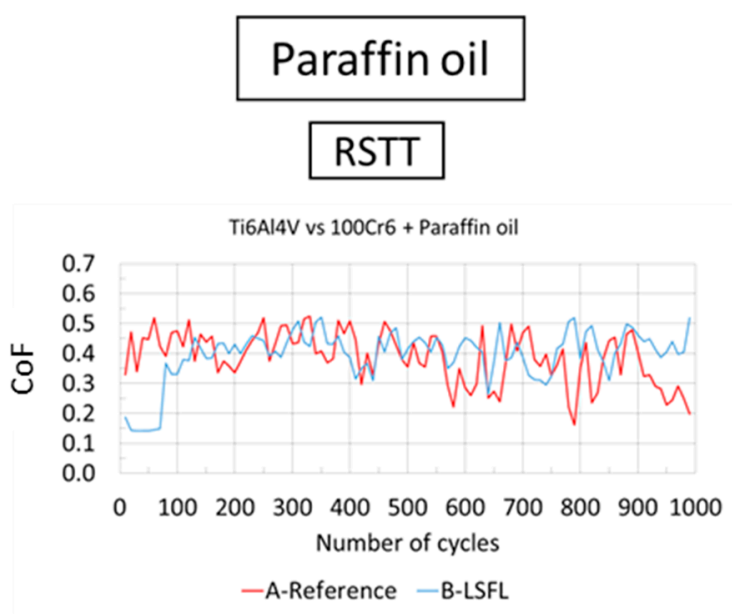

(d)

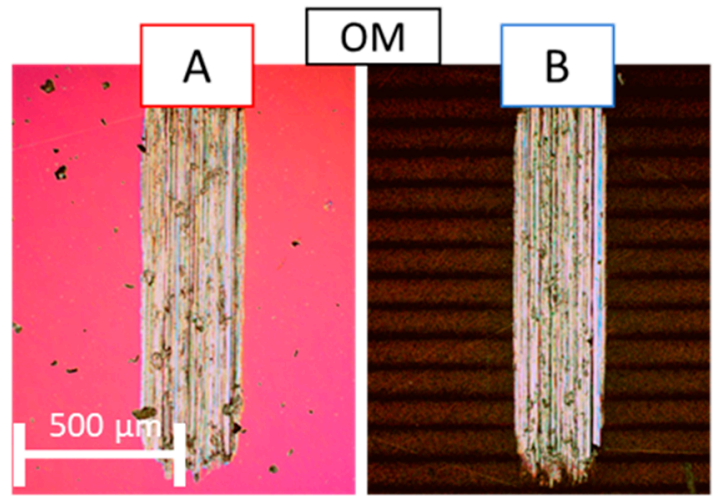

(e)

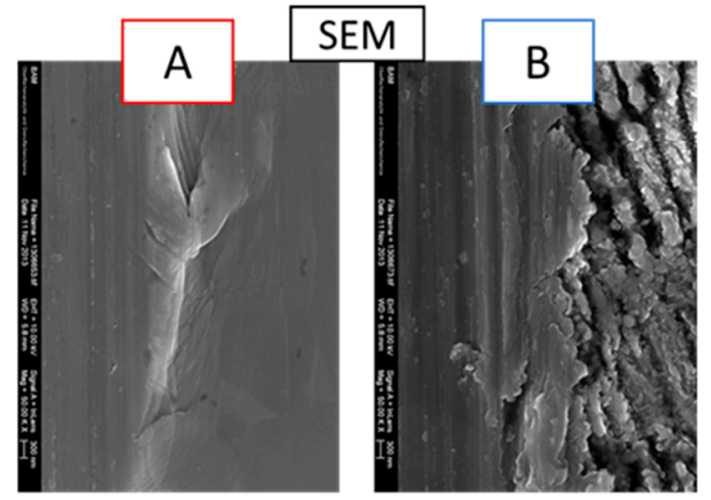

(f)

Figure 1. Tribological performance of fs-laser-processed Ti6Al4V titanium alloy after reciprocating sliding tribological tests (RSTT) against a 100Cr6 steel ball (normal force $1.0 \mathrm{~N}$, stroke $1 \mathrm{~mm}$, frequency $1 \mathrm{~Hz}$, cycles 1000). The top row exhibits the coefficient of friction $(\mathrm{CoF})$ as function of the number of sliding cycles in VPX oil (a) and paraffin oil (d). The middle row displays optical micrographs of the corresponding wear tracks after the tribological tests $(\mathbf{b}, \mathbf{e})$. The bottom row shows detailed scanning electron microscope (SEM) micrographs taken at the center (c) or at the edge (f) of the wear tracks. The data for the RSTT in paraffin oil are taken from [10]. The capitalized labels indicate tests performed on polished surfaces (A) or fs-laser-processed (low spatial frequency laser-induced periodic surface structures (LSFL)-covered) surfaces (B). Common scale bars are provided the left. 
Figure 1a presents the coefficient of friction as a function of the number of sliding cycles in VPX oil for the measurements made on the polished reference surface (red curve, A) and for tests performed in the fs-laser-processed area covered by LSFL (blue curve, B). The CoF acquired on the polished surface varies around $0.4 \pm 0.1$ through the entire test. In contrast, the CoF measured in the laser-processed region starts at a significantly lower value of $\sim 0.12 \pm 0.02$ before it sharply rises after $\sim 80$ cycles for paraffin oil and $\sim 100$ cycles for VPX oil to a similar level as seen for the reference curve. This sudden rise of the $\mathrm{CoF}$ is indicative of the starting damage of the surface when the protecting laser-induced oxide layer along with deeper lying material gets removed and the LSFL have no influence on the CoF behaviour anymore. This is in line with the visual inspections of the corresponding wear tracks shown in Figure $1 b, c$, where a severe surface damage is seen for both the polished and the laser-processed surfaces tested in VPX oil. The detailed SEM images further confirm that the LIPSS have not endured the tribological tests (compare Figure 1c, A, B). The mean values and corresponding standard deviations of the CoF and the wear volumes, quantified as $1070 \times 10^{-6} \mathrm{~mm}^{3}$ (reference) and $\sim 840 \times 10^{-6} \mathrm{~mm}^{3}$ (LSFL), are listed in Table 2. These new results obtained with VPX oil as lubricant are in line with our previous results reported for paraffin oil (compare with the right column of Figure 1).

Table 2. Mean values and standard deviations of the coefficient of friction (CoF) corresponding to the entire test (RSTT in Ti6Al4V against 100Cr6 ball, normal force $1.0 \mathrm{~N}$, stroke $1 \mathrm{~mm}$, frequency 1 $\mathrm{Hz}$, cycles 1000) and resulting wear volumes for different lubricants and tested surfaces [reference (polished) and fs-laser-processed (LSFL-covered)]. The data for the RSTT in VP1 oil and in paraffin oil are taken from [10], wear volume n.a.-not available.

\begin{tabular}{|c|c|c|c|c|}
\hline \multirow[t]{2}{*}{ Lubricant } & \multicolumn{2}{|c|}{ CoF } & \multicolumn{2}{|c|}{$\begin{array}{l}\text { Wear Volume }\left[10^{-6}\right. \\
\left.\mathrm{mm}^{3}\right]\end{array}$} \\
\hline & Reference & LSFL & Reference & LSFL \\
\hline Paraffin oil & $0.388 \pm 0.087$ & $0.395 \pm 0.087$ & n.a. & n.a. \\
\hline VPX oil & $0.391 \pm 0.044$ & $0.366 \pm 0.096$ & 1070 & 838 \\
\hline VP1 oil & $0.362 \pm 0.043$ & $0.137 \pm 0.004$ & n.a. & n.a. \\
\hline VPX oil + 0.5\% RC 3180 & $0.430 \pm 0.054$ & $0.088 \pm 0.036$ & 1039 & 0.35 \\
\hline
\end{tabular}

In order to elucidate the role of the specific additive RC 3180, identical RSTT were performed in VPX oil $+0.5 \%$ RC 3180 . These results are compared to the ones previously accomplished for the fully formulated commercial engine oil Castrol VP1, including the anti-wear additive zinc dialkyl dithiophosphate (ZDDP) [12]. Figure 2 collects the RSTT results obtained for the additivated VPX oil (left column) and our previous results for VP1 oil (right column).

Figure 2a displays the CoF vs. the number of sliding cycles in RC 3180-additivated VPX oil for the RSTT on the polished reference surface (red curve, A) along with the results obtained on the LSFL-covered surface (blue curve, B). Similar to the behaviour seen previously for VPX oil in Figure 1, the $\mathrm{CoF}$ recorded on the polished reference surface varies around $0.45 \pm 0.15$. However, the $\mathrm{CoF}$ recorded in the LSFL-covered region stays rather constant around $\sim 0.1 \pm 0.05$ through the entire test duration, with some additional spiked features between $\sim 200$ and $\sim 600$ cycles. The latter are probably caused by worn particles intermittently present in the tribological contact area. The remarkable difference in the CoF between the polished and the LSFL-covered Ti-alloy surfaces is also clearly visible in the wear tracks visualized after the RSTT by optical microscopy (OM) (Figure 2b) and SEM (Figure 2c). The SEM images confirm that the LIPSS widely endured the tribological tests (compare A, $B$ in Figure 2c). Very different wear volumes were found, estimated as $\sim 1040 \times 10^{-6} \mathrm{~mm}^{3}$ (reference) and $\sim 0.35 \times 10^{-6} \mathrm{~mm}^{3}$ (LSFL), see Table 2 . The results obtained with RC 3180-additivated VPX oil are again very similar to our previous results reported for the fully formulated VP1 oil (compare with the right column of Figure 2). Note that the use of RC 3180-additivated VPX oil results in a lower average CoF-level than that of VP1 oil, supposedly affected by improved data acquisition rate of the tribometer. Moreover, the periods of the partly worn LSFL in wear track B of Figure $2 \mathrm{c}$ are somewhat smaller than 
the ones for the corresponding track B presented in Figure 2f. This arises from somewhat differently laser fluences locally accumulated through the line processing with a Gaussian laser beam.

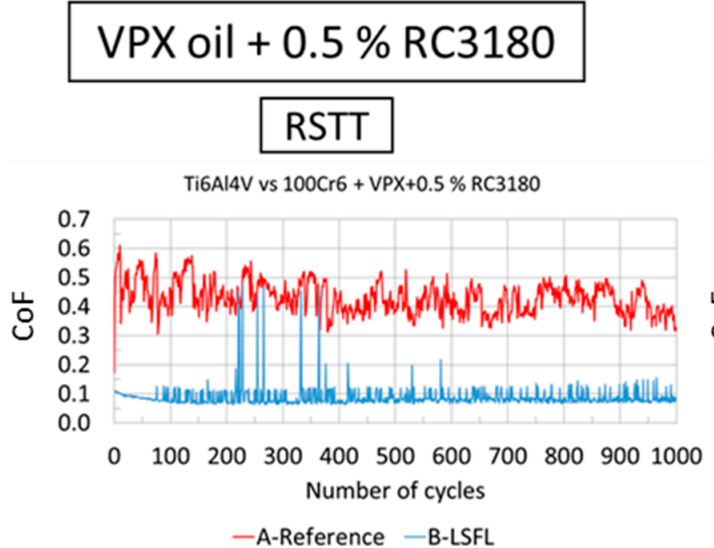

(a)

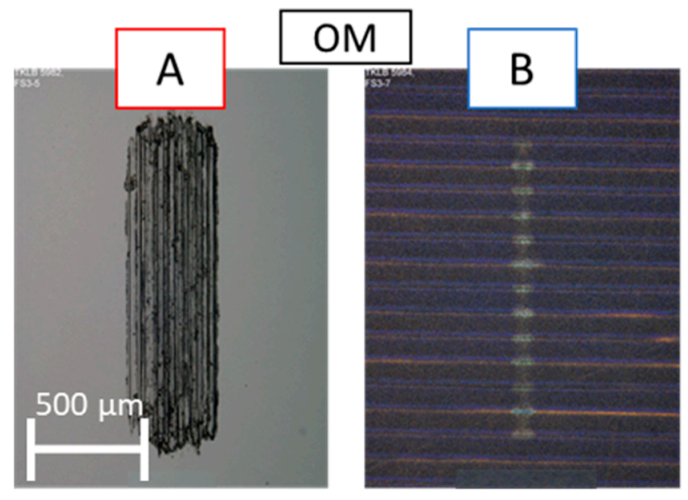

(b)

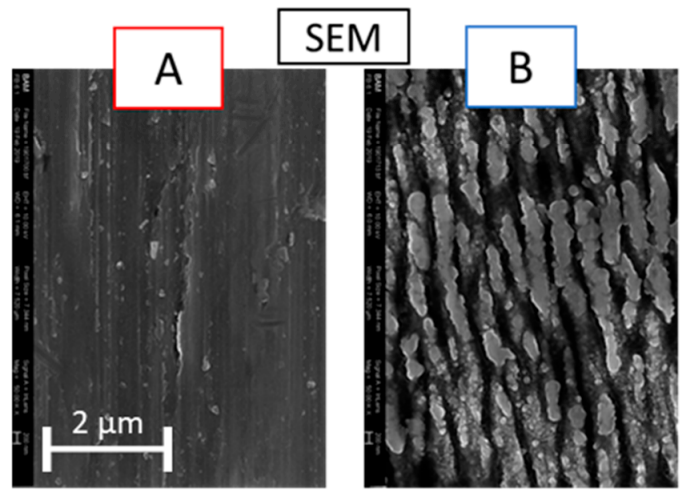

(c)

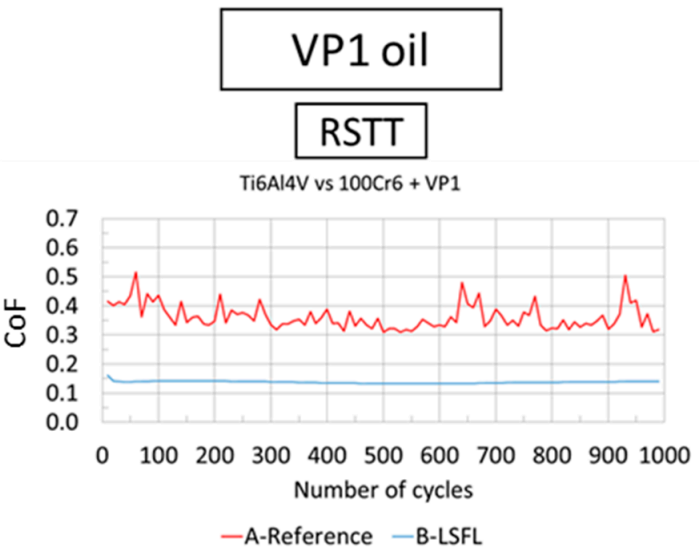

(d)

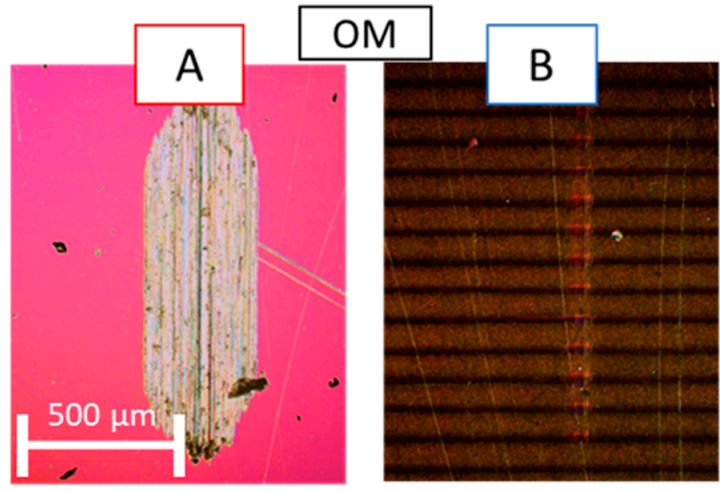

(e)

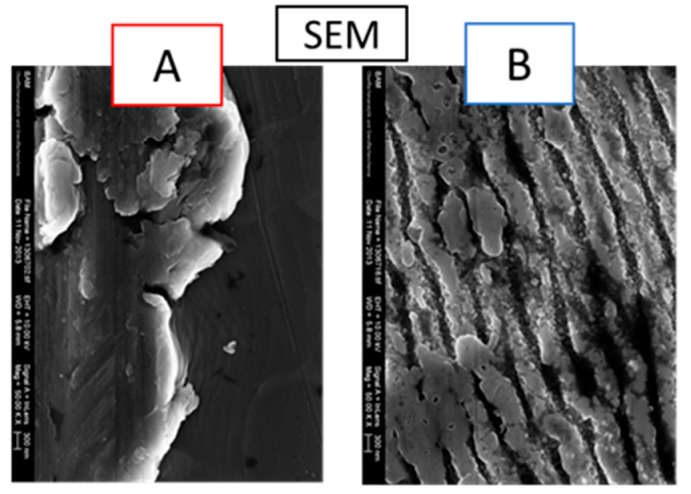

(f)

Figure 2. Tribological performance of fs-laser-processed Ti6Al4V titanium alloy after RSTT against a 100Cr6 steel ball (normal force $1.0 \mathrm{~N}$, stroke $1 \mathrm{~mm}$, frequency $1 \mathrm{~Hz}$, cycles 1000). The top row exhibits the coefficient of friction (CoF) as function of the number of sliding cycles in RC 3180-additivated VPX oil (VPX oil $+0.5 \%$ RC 3180) (a) and VP1 oil (d). The middle row displays optical micrographs of the corresponding wear tracks after the tribological tests $(\mathbf{b}, \mathbf{e})$. The bottom row shows detailed SEM micrographs taken at the center of the wear tracks $(\mathbf{c}, \mathbf{f})$. The data for the RSTT in VP1 oil are taken from [10]. The capitalized labels indicate tests performed on polished surfaces (A) or fs-laser-processed (LSFL-covered) surfaces (B). Common scale bars are provided at the left. 
Comparing the findings presented in Figures 1 and 2 for VPX oil and RC 3180-additivated VPX oil provides a direct evidence that the beneficial tribological performance featured at the fs-laser-processed surfaces covered with LSFL is caused by the anti-wear additive RC 3180 and not by their topographical characteristics. This is also seen in Figure 3, which visualizes the values compiled in Table 2. However, this improved tribological performance is likely promoted by the laser-induced graded oxide layer as it is not present for the polished Ti-alloy surface. While the roughened fs-laser-induced oxide layer on the LSFL consists mainly of amorphous $\mathrm{TiO}_{2}$ and extends $\sim 200 \mathrm{~nm}$ into depth, the native oxide layer ( $<10 \mathrm{~nm}$ thick) [13] on the smooth polished surface is too thin to interact efficiently with the 2-ethylhexyl zinc dithiophosphate molecules. It is also interesting to note that compared to the fully formulated/additivated VP1 oil, this beneficial reduction in CoF and wear can already be achieved by solely using RC 3180.

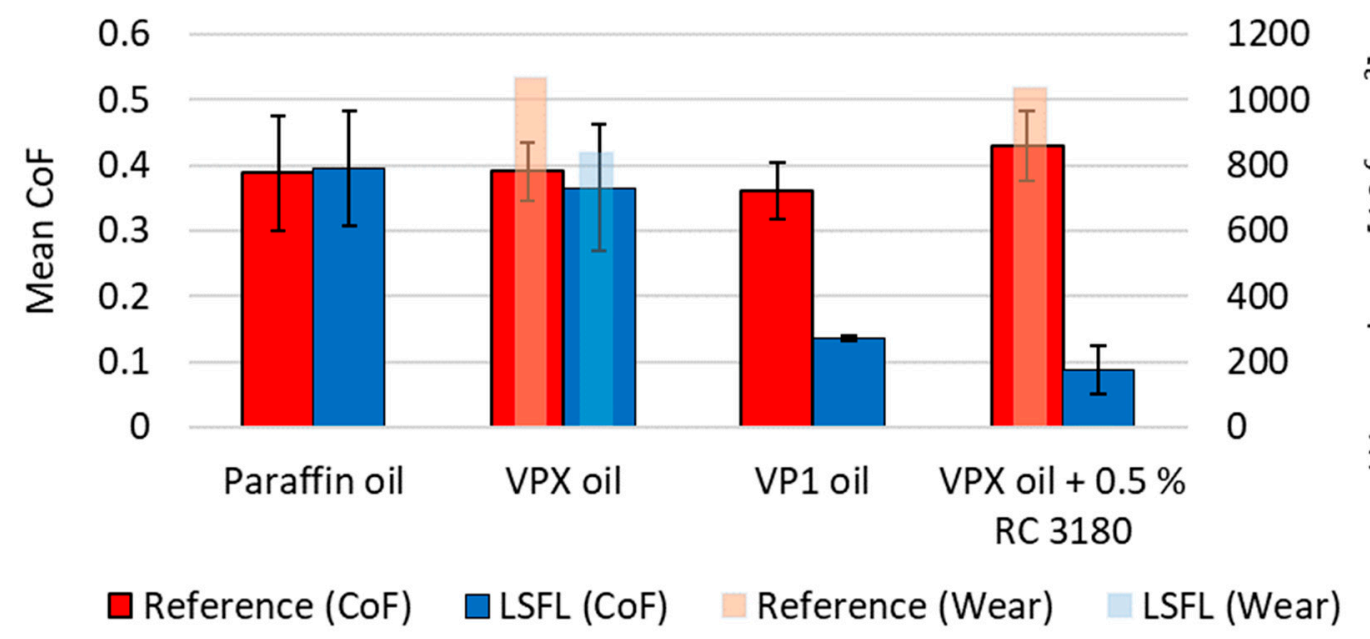

Figure 3. Visualization of the data compiled in Table 2.

It is worth noting that the initial values of the CoF in the LSFL-covered regions are all comparable at a level of 0.10-0.15 indicating the presence of a protecting oxide layer during the first hundred sliding cycles (see Figure 1a,d and Figure 2a,d). For the lubricants without anti-wear additives (paraffin oil, VPX oil), the oxide layer is worn, while with such additives (VP1 oil, VPX oil $+0.5 \%$ RC 3180 ) the laser-induced oxide layer endures the entire tribological test.

For a deeper characterization of a wear track obtained with an anti-wear additive, we extended our previous STEM analysis [14] by adding spatially information on the elemental contribution underneath the laser-irradiated and worn surface. The corresponding wear track was obtained in the same processed LSFL area as previously shown in Figure $2 \mathrm{~d}, \mathrm{e}(\mathrm{B}), \mathrm{f}(\mathrm{B})$, but it was tribologically tested parallel to the ablation lines, i.e., perpendicular to the LSFL, with otherwise unchanged RSTT conditions. Figure 4a shows an optical micrograph of the worn surface after an RSTT on LSFL-covered titanium alloy surfaces in VP1 oil. Note that the dark vertical lines arise from the line wise laser processing, while the bright lines in the centre correspond to the wear track. From this tribologically tested surface, a FIB lamella was prepared. The location it was taken from is marked in green in Figure $4 \mathrm{~b}$. A cross-sectional STEM image was taken in bright field mode from this lamella, which is presented in Figure 4c. The wear track is marked with a black double arrow. The depth of the zone affected by the laser structuring process and tribological testing can be estimated to be less than $500 \mathrm{~nm}$. This zone is due to the local chemistry in the tribological contact area that creates a protective surface layer in the LSFL. It prevents a direct contact between the two sliding bodies, resulting in reduced friction and wear (Figure 2). The STEM image clearly shows the grain structure of the polycrystalline Ti6Al4V alloy. In all images, the protective Pt layer is marked, which consists of two sublayers. Figure $4 \mathrm{~d}$ is a magnified detail of Figure 4c, of which elemental maps (EDX) were established for Fe (Figure 4e), Ti (Figure 4f), and Zn (Figure 4g). Signals in the top part of these images are reflections of the Pt protective 
layer. Close to the Ti surface, material transfer (Fe) of the steel counterbody is visible (Figure 4e), as well as Zn (Figure 4g) of the ZDDP additive. Figure 4 h shows a superposition of the STEM image (Figure $4 \mathrm{~d}$ ) with the elemental maps of Fe, Ti, and Zn. It reveals that within the wear track, small pits have formed, which contain more or less loose debris. In these locations, Zn could be found, which is an indicator for the presence of ZDDP.
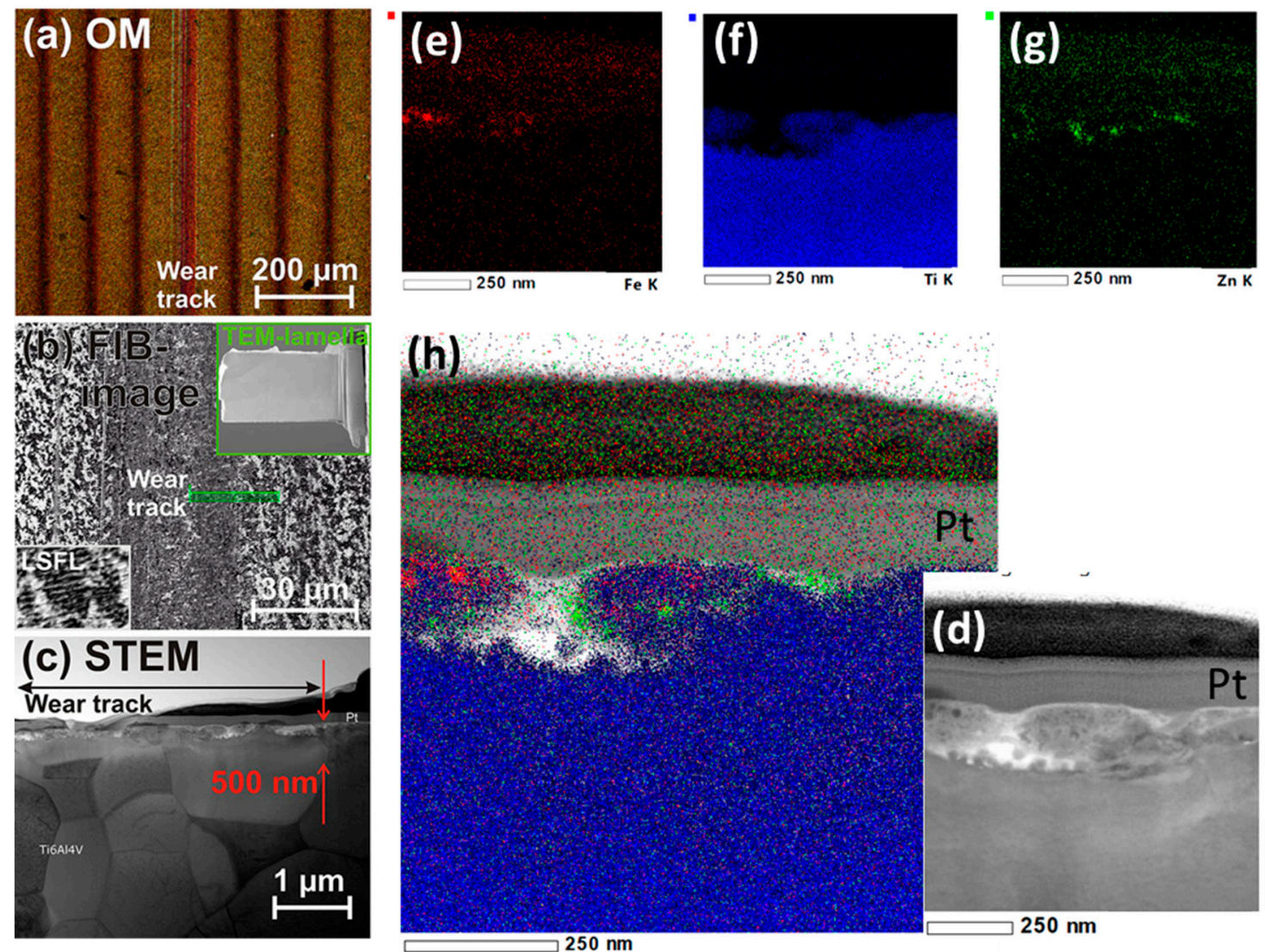

Figure 4. Characterization of a wear track on LSFL-covered Ti4Al6V after RSTT [identical processed LSFL area as previously shown in Figure 2e(B), but tribologically tested parallel to the ablation lines (perpendicular to the LSFL) with otherwise unchanged RSTT conditions]. (a) optical micrograph. (b) Focused ion beam (FIB) image with respective lamella and magnified detail. (c) STEM cross-sectional image of this lamella. (d) magnified detail of (c), of which energy-dispersive X-ray (EDX) spectroscopy elemental maps were taken for $\mathrm{Fe}(\mathbf{e}), \mathrm{Ti}(\mathbf{f})$ and $\mathrm{Zn}(\mathbf{g}),(\mathbf{h})$ is an overlay of $(\mathbf{d}-\mathbf{g})$. Images $(\mathbf{a}-\mathbf{c})$ are taken from [14].

\subsection{RSTT against Different Counterbody Materials}

The additives ZDDP are highly optimized to work efficiently on metallic (mostly steel) surfaces as anti-wear agents [12,17-19]. Since in the previous experiments (shown in Section 3.1) balls made of $100 \mathrm{Cr} 6$ steel were used as counterbodies, the question remains open, whether the beneficial tribological effect originates from the binding of the additive molecules to the oxidized titanium alloy surface or to the surface of the steel ball. Hence, in a second set of experiments the material of the counterbody was systematically varied in order to use also non-metallic, i.e., oxide- $\left(\mathrm{Al}_{2} \mathrm{O}_{3}\right)$ or nitride-based $\left(\mathrm{Si}_{3} \mathrm{~N}_{4}\right)$ ceramics, which feature different chemical compositions and were available in a similar surface quality as the 100Cr6 steel balls.

Figure 5 gathers the results by showing the graphs of the CoF and OM and SEM micrographs of the corresponding wear tracks on polished and fs-laser-processed Ti6Al4V surface after the RSTT in VPX oil $+0.5 \%$ RC 3180 against balls made of $100 \mathrm{Cr} 6$ steel [left column, (a)], $\mathrm{Al}_{2} \mathrm{O}_{3}$ ceramic [middle column, (b)] and $\mathrm{Si}_{3} \mathrm{~N}_{4}$ ceramic [right column, (c)]. 

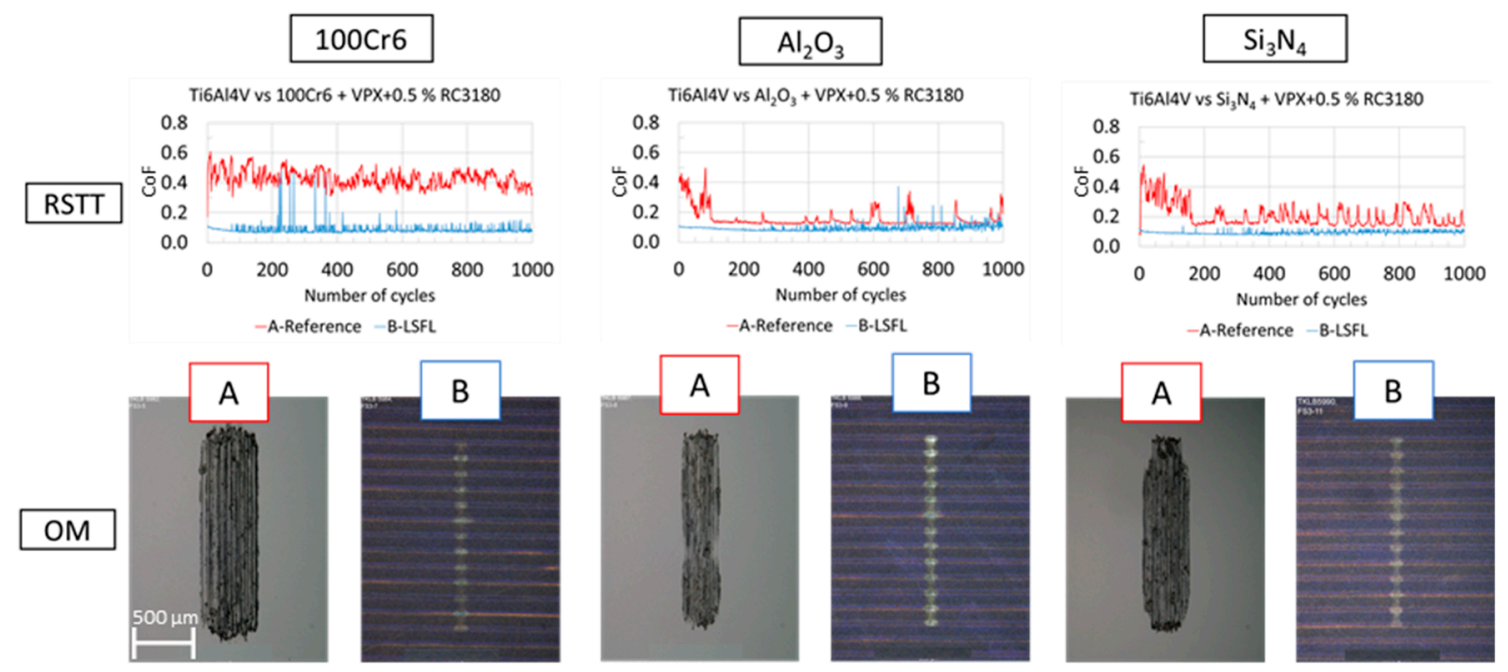

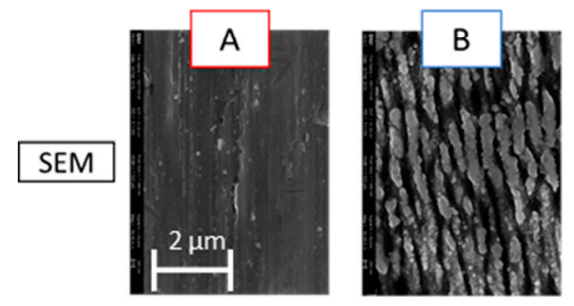

(a)

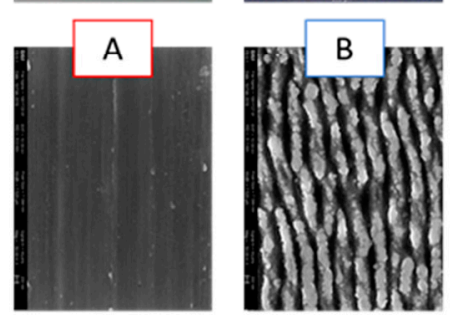

(b)

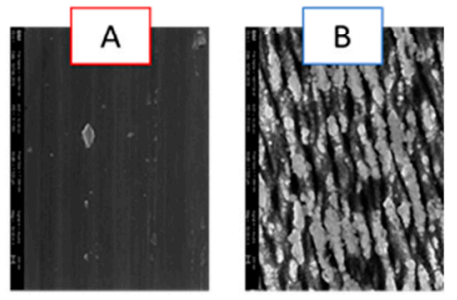

(c)

Figure 5. Tribological performance of fs-laser-processed Ti6Al4V surfaces after RSTT (normal force $1.0 \mathrm{~N}$, stroke $1 \mathrm{~mm}$, frequency $1 \mathrm{~Hz}$, cycles 1000) in RC 3180-additivated VPX oil (VPX oil + 0.5\% RC 3180) against $10 \mathrm{~mm}$ balls made of different materials. RSTT against 100Cr6 steel (metallic) counterbody (a), $\mathrm{Al}_{2} \mathrm{O}_{3}$ (oxide ceramic) counterbody (b) and $\mathrm{Si}_{3} \mathrm{~N}_{4}$ (non-oxide ceramic) counterbody (c). The top row provides the coefficient of friction $(\mathrm{CoF})$ as function of the number of sliding cycles. The middle row shows optical micrographs of the corresponding wear tracks after the tribological tests and the bottom row depicts detailed scanning electron microscope (SEM) micrographs taken at the centre of the wear tracks. The capitalized labels indicate tests performed on polished surfaces (A) or fs-laser-processed (LSFL-covered) surfaces (B). Common scale bars are provided at the left.

For a direct comparison, Figure 5a displays again the results obtained for the 100Cr6 steel balls previously shown in Figure 2 (left column). The CoF's measured in the fs-laser-processed regions (B, blue curves) with both ceramic counterbodies $\left(\mathrm{Al}_{2} \mathrm{O}_{3}, \mathrm{Si}_{3} \mathrm{~N}_{4}\right)$ are as small as those obtained for the metallic steel balls, see Table 3. In all cases, the LSFL endured the RSTT, see the high-resolution SEM images for the associated wear tracks (B) in Figure 5, bottom row. The CoF's recorded in the polished reference surfaces (A, red curves) always start between $\sim 0.4-0.6$. However, after less than 200 cycles the CoF's are remarkably lower for the ceramic counterbodies when compared to the metallic steel ball. Nevertheless, the averaged CoF always shows a clear reduction between the polished surfaces and the fs-laser-processed regions, although the reduction ratio is smaller for both ceramic counterbodies. The reduced CoF's on the polished surfaces when using ceramic balls compared to the steel balls are also reflected in the size of the corresponding wear tracks (compare the tracks A in the OM, middle row, Figure 5). 
Table 3. Mean values and standard deviations of the coefficient of friction ( $\mathrm{CoF})$ corresponding to the entire test (RSTT in Ti6Al4V, normal force $1.0 \mathrm{~N}$, stroke $1 \mathrm{~mm}$, frequency $1 \mathrm{~Hz}$, cycles 1000) and resulting wear volumes for different lubricants and tested surfaces [reference (polished) and fs-laser-processed (LSFL-covered)] using different counterbody materials.

\begin{tabular}{cccccc}
\hline \multirow{2}{*}{ Lubricant } & \multirow{2}{*}{ Counterbody } & \multicolumn{2}{c}{ CoF } & \multicolumn{2}{c}{ Wear Volume $\left[\mathbf{1 0}^{-\mathbf{6}} \mathbf{~ m m}^{\mathbf{3}}\right]$} \\
\cline { 3 - 6 } & & Reference & LSFL & Reference & LSFL \\
\hline \multirow{2}{*}{ VPX oil } & $100 \mathrm{Cr6}$ & $0.391 \pm 0.044$ & $0.366 \pm 0.096$ & 1070 & 838 \\
& $\mathrm{Al}_{2} \mathrm{O}_{3}$ & $0.167 \pm 0.053$ & $0.148 \pm 0.078$ & 175 & 295 \\
VPX oil + 0.5\% & $100 \mathrm{Cr} 6$ & $0.430 \pm 0.054$ & $0.088 \pm 0.036$ & 1039 & 0.35 \\
RC 3180 & $\mathrm{Al}_{2} \mathrm{O}_{3}$ & $0.158 \pm 0.063$ & $0.094 \pm 0.022$ & 169 & 7 \\
& $\mathrm{Si}_{3} \mathrm{~N}_{4}$ & $0.206 \pm 0.077$ & $0.094 \pm 0.012$ & 452 & 4 \\
\hline
\end{tabular}

The quantitative values of the averaged $\mathrm{CoF}^{\prime}$ s along with the estimated wear volumes provided in Table 3 prove that the difference in the counterbody material (chemical composition as well as hardness) and the tribological contact characteristics estimated from an elastic (Hertzian) deformation model [20] (contact pressure, contact radius, sample-ball deformation, see Table 1) are not the dominating effect in our RSTT featuring the beneficial tribological effect. Hence, it is likely that the laser-induced oxidation and the interplay with surface topography are the crucial aspects here. Inferring that a minimum oxygen-containing layer thickness and an enlarged surface area are required together with the additives ZDDP [14] to form a sufficiently thick anti-wear layer during RSTT, which prevents a direct contact of the two sliding tribological bodies. These findings are supported by Figure 6, which visualizes the values gathered in Table 3.

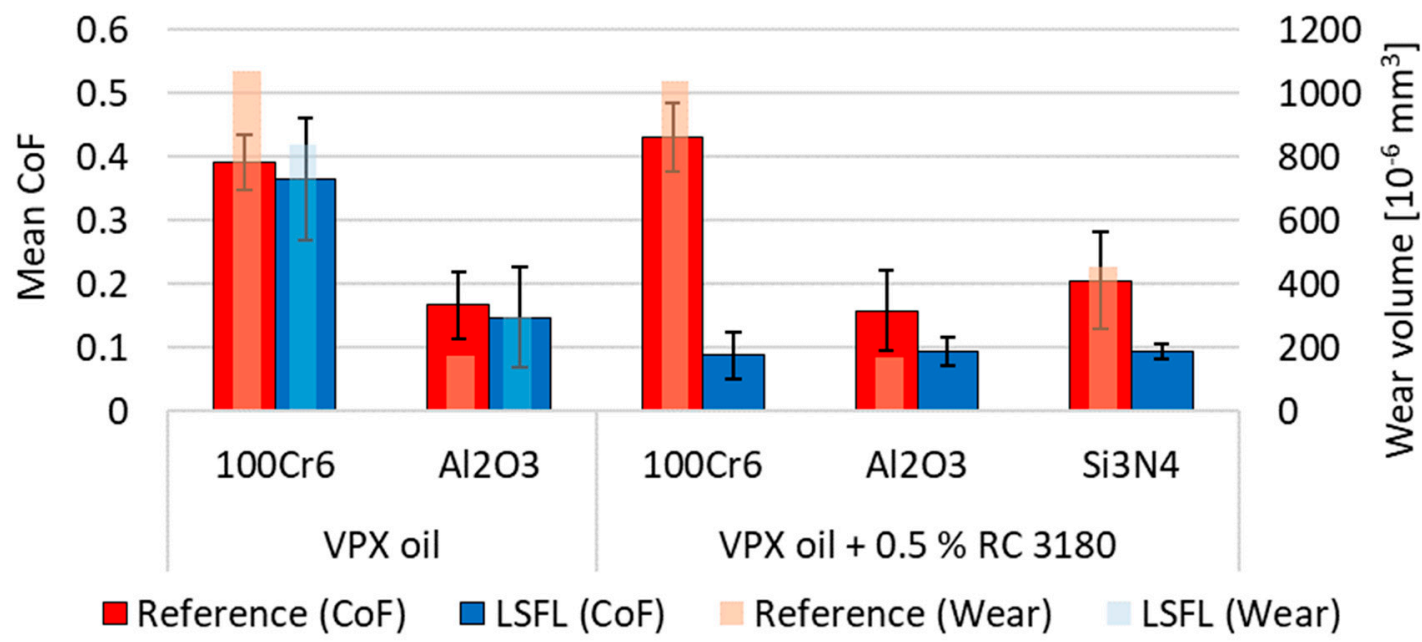

Figure 6. Visualization of the data compiled in Table 3.

\section{Conclusions}

Low spatial frequency LIPSS (LSFL) were uniformly processed by Ti:sapphire fs-laser pulses on Ti6Al4V titanium alloy samples. The tribological performance of the surfaces [reference (polished) vs. fs-laser-processed (LSFL-covered)] was determined in linear reciprocating sliding tribological tests against $100 \mathrm{Cr} 6, \mathrm{Al}_{2} \mathrm{O}_{3}$ and $\mathrm{Si}_{3} \mathrm{~N}_{4}$ balls as counterbodies in two different lubricants [VPX oil and VPX oil $+0.5 \%$ of an anti-wear (ZDDP) additive, RC 3180]. Subsequently, the wear tracks were characterized by OM, SEM, STEM, and confocal profilometry. For the specific testing conditions here, a reduction by a factor of $4-5$ for the coefficient of friction and by a factor $>2500$ for the wear volume was observed in RC 3180-additivated VPX oil, while the tribological tests against 100Cr6 balls showed no beneficial influence of LSFL-covered surfaces with VPX oil lubrication. These new results proved the similar behaviour between VPX oil and paraffin oil (free of any additives) and between RC 
3180-additivated VPX oil and commercial VP1 oil containing ZDDP as anti-wear agent. This clearly evidences our previous speculation that only the admixture of the specific ZDDP molecules account for the reproduction of the beneficial tribological behaviour on the laser-processed Ti6Al4V surfaces. Additionally, no significant influence of the counterbody material (metal vs. ceramics) was observed, thereby implying that the positive effect is mainly caused by the presence of the 2-ethylhexyl zinc dithiophosphate molecules in the lubricant along with a nanostructured and oxidized layer on the laser-processed surfaces. The interplay between the sample topography (featuring an enlarged surface area and a confinement of the lubricant) and the local chemistry in the tribological contact area-via formation of a protective surface layer on the LSFL—prevents a direct contact of the two sliding bodies, finally resulting in reduced friction and wear.

Author Contributions: Conceptualization, D.S. and J.B.; Methodology, J.B. and D.S.; Validation, all authors; Formal analysis, J.J.A. and N.S.; Investigation, all authors; Resources, J.B., N.S., and D.S.; Data curation, J.J.A. and N.S.; Writing—original draft preparation, J.J.A. and J.B.; Writing—review and editing, all authors; Visualization, J.J.A., N.S., and J.B.; Supervision, D.S., J.B., A.Z., I.L., and A.A.; Project administration, D.S., J.B., A.Z., and I.L.; Funding acquisition, J.J.A., A.Z., J.B. and D.S.

Funding: This work was supported by the ERASMUS+ program of the European Union (KA103). This project has received funding from the European Union's Horizon 2020 research and innovation programme under grant agreement No. 814494 (project "i-TRIBOMAT") and grant agreement No. 665337 (project "LiNaBioFluid"). The Surface Technologies research group (Mondragon University, Faculty of Engineering) gratefully acknowledges the financial support given by the Red Guipuzcoana de Ciencia Tecnología e Innovación 2018 program through the project ASEFI (Orden Foral Número 218/2018).

Acknowledgments: The authors would like to thank C. Neumann (BAM 6.3) for help with profilometric sample characterizations, S. Benemann (BAM 6.1) for the SEM characterizations, R. Koter (BAM 6.4) for the laser surface processing of a sample copy, and S. Binkowski (BAM 6.3) for polishing the titanium samples. The STEM analysis was performed by I. Dörfel (BAM 5.1). The femtosecond laser processing was performed within the frame of German Science Foundation (Deutsche Forschungsgemeinschaft, DFG) project under Grant KR 3638/1-2.

Conflicts of Interest: The authors declare no conflict of interest.

\section{References}

1. Abere, M.; Zhong, M.; Krüger, J.; Bonse, J. Ultrafast laser-induced morphological transformations. MRS Bull. 2016, 41, 969-974. [CrossRef]

2. Liu, H.; Lin, W.; Hong, M. Surface coloring by laser irradiation of solid substrates. APL Photonics 2019, 4, 051101. [CrossRef]

3. Kirner, S.V.; Hermens, U.; Mimidis, A.; Skoulas, E.; Florian, C.; Hischen, F.; Plamadeala, C.; Baumgartner, W.; Winands, K.; Mescheder, H.; et al. Mimicking bug-like surface structures and their fluid transport produced by ultrashort laser pulse irradiation of steel. Appl. Phys. A 2017, 123, 754. [CrossRef]

4. $\quad$ Epperlein, N.; Menzel, F.; Schwibbert, K.; Koter, R.; Bonse, J.; Sameith, J.; Krüger, J.; Toepel, J. Influence of femtosecond laser produced nanostructures on biofilm growth on steel. Appl. Surf. Sci. 2017, 418, 420-424. [CrossRef]

5. Heitz, J.; Plamadeala, C.; Muck, M.; Armbruster, O.; Baumgartner, W.; Weth, A.; Steinwender, C.; Blessberger, H.; Kellermair, J.; Kirner, S.V.; et al. Femtosecond laser-induced microstructures on Ti substrates for reduced cell adhesion. Appl. Phys. A 2017, 123, 734. [CrossRef]

6. Bonse, J.; Kirner, S.V.; Griepentrog, M.; Spaltmann, D.; Krüger, J. Femtosecond laser texturing of surfaces for tribological applications. Materials 2018, 11, 801. [CrossRef] [PubMed]

7. Bäuerle, D. Laser Processing and Chemistry, 4th ed.; Springer: Berlin/Heidelberg, Germany, 2011.

8. Bonse, J.; Höhm, S.; Kirner, S.V.; Rosenfeld, A.; Krüger, J. Laser-induced periodic surface structures-A scientific evergreen. IEEE J. Sel. Top. Quantum Electron. 2017, 23, 9000615. [CrossRef]

9. Bonse, J.; Höhm, S.; Koter, R.; Hartelt, M.; Spaltmann, D.; Pentzien, S.; Rosenfeld, A.; Krüger, J. Tribological performance of sub-100-nm femtosecond laser-induced periodic surface structures on titanium. Appl. Surf. Sci. 2016, 374, 190-196. [CrossRef]

10. Bonse, J.; Koter, R.; Hartelt, M.; Spaltmann, D.; Pentzien, S.; Höhm, S.; Rosenfeld, A.; Krüger, J. Femtosecond laser-induced periodic surface structures on steel and titanium alloy for tribological applications. Appl. Phys. A 2014, 117, 103-110. [CrossRef] 
11. Bonse, J.; Koter, R.; Hartelt, M.; Spaltmann, D.; Pentzien, S.; Höhm, S.; Rosenfeld, A.; Krüger, J. Tribological performance of femtosecond laser-induced periodic surface structures on titanium and a high toughness bearing steel. Appl. Surf. Sci. 2015, 336, 21-27. [CrossRef]

12. Nicholls, M.A.; Do, T.; Norton, P.R.; Kasrai, M.; Bancroft, G.M. Review of the lubrication of metallic surfaces by zinc dialkyl-dithiophosphates. Tribol. Int. 2005, 38, 15-39. [CrossRef]

13. Kirner, S.V.; Wirth, T.; Sturm, H.; Krüger, J.; Bonse, J. Nanometer-resolved chemical analyses of femtosecond laser-induced periodic surface structures on titanium. J. Appl. Phys. 2017, 122, 104901. [CrossRef]

14. Kirner, S.V.; Slachciak, N.; Elert, A.M.; Griepentrog, M.; Fischer, D.; Hertwig, A.; Sahre, M.; Dörfel, I.; Sturm, H.; Pentzien, S.; et al. Tribological performance of titanium samples oxidized by fs-laser radiation, thermal heating, or electrochemical anodization. Appl. Phys. A 2018, 124, 326. [CrossRef]

15. Klaffke, D.; Hartelt, M. Tribological characterization of thin hard coatings by reciprocating sliding tests. Tribol. Lett. 1995, 1, 265-276. [CrossRef]

16. ASTM D7755-11 2017. Standard Practice for Determining the Wear Volume on Standard Test Pieces Used by High-Frequency, Linear-Oscillation (SRV) Test Machine; ASTM: West Conshohocken, PA, USA, 2017.

17. Itoa, K.; Martin, J.M.; Minfray, C.; Katoa, K. Low-friction tribofilm formed by the reaction of ZDDP on iron oxide. Tribol. Int. 2006, 39, 1538-1544. [CrossRef]

18. Qu, J.; Blau, P.J.; Howe, J.Y.; Meyer, H.M. Oxygen diffusion enables anti-wear boundary film formation on titanium surfaces in zinc-dialkyl-dithiophosphate (ZDDP)-containing lubricants. Scipta Mater. 2009, 60, 886-889. [CrossRef]

19. Hsu, C.; Stratmann, A.; Rosenkranz, A.; Gachot, C. Enhanced growth of ZDDP-based tribofilms on laser-interference patterned cylinder roller bearings. Lubricants 2017, 5, 39. [CrossRef]

20. Stachowiak, G.W.; Batchelor, A.W. Engineering Tribology; Tribology Series 24; Elsevier: Amsterdam, The Netherlands, 1993; p. 349.

(C) 2019 by the authors. Licensee MDPI, Basel, Switzerland. This article is an open access article distributed under the terms and conditions of the Creative Commons Attribution (CC BY) license (http://creativecommons.org/licenses/by/4.0/). 